\title{
Experiencias
}

\section{Tecnología, creatividad y reinvención de actividades: las claves del éxito en la atención educativa a alumnos con discapacidad visual en tiempos de pandemia}

Technology, creativity and reinvention of activities: keys to successfully educate students with visual disability during a pandemic

\section{S. Boix Hernández, M. T. Corbella Roqueta, F. Torres Portero}

\section{Resumen}

Este artículo muestra la experiencia del Centro de Recursos de la ONCE en BarceIona durante la pandemia de la covid-19: la aceleración del cambio tecnológico, el proceso de adaptación y transformación de sus actividades para seguir atendiendo a los alumnos con discapacidad visual, a sus familias y a los profesionales de su entorno escolar. Después de una breve mención de los cambios provocados en los procesos de comunicación (de presencial a virtual), la creación de espacios colaborativos en la nube y el cambio en los procesos de atención telemática, nos centraremos en la exposición de algunas actividades del Centro que han pasado de presenciales a telemáticas, en los inconvenientes y las soluciones aportadas, así como en el feedback de los usuarios (alumnos, profesionales y familias). Una experiencia positiva, pero en continua evolución y proceso de mejora.

\section{Palabras clave}

Covid-19. Discapacidad visual. Tecnología. Aprendizaje virtual. Atención telemática. 


\begin{abstract}
This article discusses an experience conducted by the ONCE Educational Resource Centre at Barcelona during the covid-19 pandemic. Involved were expedition of technological change and adaptation and transformation of centre activities to continue to provide support for students with visual disability, as well as for their families and teachers. A brief review of the changes in communication (from in-person to virtual) and ICTbased support and of the creation of cloud-based collaborative spaces is followed by a description of the redesign of centre activities for delivery via ICT. The setbacks arising, solutions found and user (student, teacher and family) feedback are also addressed. Whilst the experience has proved beneficial, it requires ongoing development and improvement.
\end{abstract}

\title{
Key words
}

Covid-19. Visual disability. Technology. Virtual learning. ICT-mediated care.

\section{Introducción}

La pandemia provocada por la covid-19 ha supuesto un antes y un después en muchos campos. Sin lugar a duda, ha impulsado el uso de la tecnología en toda la sociedad: formación a distancia, consultas médicas, teletrabajo, compras, reuniones telemáticas, cultura, etc.

La transformación digital se ha acelerado y ha llegado a muchas personas que nunca hubieran imaginado que usarían la tecnología en su día a día.

A pesar de los inconvenientes que ha supuesto la aplicación forzada de muchos de estos cambios (falta de formación tecnológica, falta de recursos, falta de accesibilidad de los contenidos, aislamiento, etc.), se puede pensar también en las oportunidades que ha generado esta situación.

En nuestro caso, desde el Centro de Recursos Educativos de la ONCE en Barcelona (CREDV-CRE ONCE Barcelona), cuyo objetivo principal es la atención directa a alumnos con discapacidad visual, a sus familias y a los profesionales de su entorno educativo, la pandemia ha provocado un cambio en muchos aspectos:

Boix, S., Corbella, M.T., y Torres, F. (2021). Tecnología, creatividad y reinvención de actividades: las claves del éxito en la atención educativa a alumnos con discapacidad visual en tiempos de pandemia. RED Visual: Revista Especializada en Discapacidad Visual, 77, 240-263. https://doi.org/10.53094/LWAR1212. 
- Revisión y desarrollo de nuevos procesos de comunicación. Videoconferencias entre profesionales, profesor-alumno, maestro de soportefamilias... en las que, aparte de los aspectos más técnicos y formativos del uso de las herramientas, se han definido los parámetros de enseñanza virtual, las estrategias y criterios de atención en el caso de alumnos con discapacidad visual.

- Creación y desarrollo de espacios de colaboración y recursos para compartir experiencias y atender las necesidades de los maestros de soporte. Estos espacios colaborativos han permitido la recogida de materiales digitales y la potenciación del interés por compartir recursos, formaciones, etc.

- Desarrollo de nuevos procesos y metodologías de atención a las familias y alumnos en el caso de atención precoz, educación infantil y alumnos en general que todavía no son autónomos en el uso de las tecnologías.

- Reinvención de las actividades de formación dirigidas a profesionales internos y externos, a alumnos y a familias para el curso 2020-2021. Seminarios de formación de las distintas áreas de especial dificultad (accesibilidad y tecnología, plástica, inglés, música, matemáticas, deportes...) y presentación del CREDVCRE ONCE Barcelona como servicio educativo a los nuevos tutores, actividades enmarcadas en el Servicio de Escolaridad Combinada (SEC), ya sean individuales o grupales (actividades complementarias).

Dichos cambios en los procesos de intervención del CREDV-CRE ONCE Barcelona han ido acompañados de un cambio de actitud de sus profesionales con respecto a la tecnología, un esfuerzo de superación y adaptación a la nueva situación, un deseo de atender, por encima de todo, a los alumnos con discapacidad visual y a sus familias. En definitiva, un espíritu proactivo en pos de superar la adversidad de la pandemia, ofreciendo la máxima calidad en el servicio.

Esta rápida adaptación se ha visto facilitada por la apuesta del CREDV-CRE ONCE Barcelona por el uso de las tecnologías con una estrategia de planificación y visión de futuro que se ha fundamentado en tres pilares: el uso de plataformas digitales, la formación en tecnologías de la información y la comunicación (TIC) de sus profesionales y el conocimiento de las necesidades en aprendizaje TIC de los alumnos con discapacidad visual que se atienden en este Centro. 


\section{Revisión y desarrollo de los nuevos procesos de comunicación. Videoconferencias con alumnos}

Son ya conocidas las pautas y orientaciones que se dan al maestro de aula ordinaria para dar las clases presenciales en el caso de tener alumnos con discapacidad visual. Así, debe verbalizar cuanto escribe en la pizarra, realizar descripciones de las imágenes, modelar o acompañar las manos del alumno con ceguera para recorrer un objeto, una lámina en relieve, etc. La comunicación presencial maestro-alumno con discapacidad visual está definida y además siempre disponemos del feedback inmediato del alumno con sus gestos, la expresión de su cara, etc.

La comunicación a través de videoconferencia es muy distinta. El maestro de aula no tiene al alumno con discapacidad visual en la primera fila, quizás el alumno no tiene la cámara activada, no conoce las teclas para acceder al chat, activar-desactivar el micrófono, no puede acceder a la presentación cuando el maestro comparte la pantalla... En definitiva, es necesario definir unas orientaciones para la comunicación en el caso de videoconferencias si tenemos alumnos con discapacidad visual en el aula.

Se trata de unas pautas generales independientes de la plataforma de comunicación utilizada (Google Meet, Zoom, Teams, Jitsi, etc.):

1. Nos presentamos. Dado que en la mayoría de las ocasiones se cierran los micrófonos en las videoconferencias con alumnos para evitar interrupciones, es necesario que el alumno con discapacidad visual sepa quiénes participan en la sesión. Es aconsejable presentarnos, recordar quiénes somos cuando tenemos el turno de palabra, etc.

2. En la impartición de la clase.

a. Más que nunca debemos potenciar la verbalización de la información, ya que todo es visual (una proyección) y los alumnos con discapacidad visual o con ceguera tienen dificultades o no pueden acceder a ella.

b. Se ha de utilizar un lenguaje claro, preciso y descriptivo de la información.

Boix, S., Corbella, M.T., y Torres, F. (2021). Tecnología, creatividad y reinvención de actividades: las claves del éxito en la atención educativa a alumnos con discapacidad visual en tiempos de pandemia. RED Visual: Revista Especializada en Discapacidad Visual, 77, 240-263. https://doi.org/10.53094/LWAR1212. 
c. Es imprescindible anticipar los documentos o materiales que se van a explicar en la sesión de clase (texto en Word, PowerPoint, etc.); de este modo el alumno con discapacidad visual puede disponer de ellos en el ordenador o en la tableta.

d. Es imprescindible asegurar que los documentos digitales son accesibles al revisor de pantalla y que cumplen los criterios visuales de accesibilidad.

3. Seguimiento de la sesión.

a. Formular preguntas al alumno con discapacidad visual y hacerlo partícipe durante la sesión. Quizás no puede o no sabe acceder al chat, pedir el turno de palabra, etc.

b. Asegurarse de que ha accedido a la información de forma satisfactoria y que ha comprendido las tareas encomendadas.

4. Presentación de tareas (compartir pantalla).

a. Hay que confirmar que sabe cómo compartir pantalla. Será importante darle una formación previa.

b. Respetar su ritmo de trabajo, ajustando el nivel de exigencia.

c. Ofrecerle nuestra ayuda si tiene dificultades durante la sesión.

Además de estas breves pautas, destacamos la necesidad de utilizar buenos equipos técnicos de cámara, micrófono y wifi, ya que la calidad de la imagen y del sonido tienen importancia en la recepción de la información.

También se aconseja que el maestro de aula se conecte simultáneamente con el alumno con discapacidad visual mediante control remoto (con el programa TeamViewer); de este modo, si surge algún problema durante la actividad, puede intervenir directamente y ayudarlo en su resolución. 


\section{Creación y desarrollo de espacios de colaboración y recursos}

El cambio súbito de un aprendizaje presencial a un aprendizaje telemático de todos los alumnos con discapacidad visual atendidos por el CREDV-CRE ONCE Barcelona provocó un incremento en el uso de materiales digitales, en la necesidad de analizar la accesibilidad de estos, de realizar adaptaciones, etc.

¿Cómo ser eficaces y prácticos en la creación y adaptación de materiales digitales accesibles?, ¿qué materiales son accesibles para los alumnos que trabajan con revisor de pantalla?... En este apartado desarrollamos las soluciones encontradas.

Durante el confinamiento, decidimos aprovechar la plataforma Moodle de la Generalitat y los servicios de Google como lugar de encuentro y colaboración entre los distintos profesionales. Iniciamos un proyecto colaborativo de recopilación de adaptaciones y recursos digitales accesibles que cada maestro iba elaborando para la atención de los alumnos con los que intervenía.

Cada equipo de trabajo creó un espacio en Google Drive accesible a todos sus miembros y, a su vez, lo compartía con todos los profesionales del CRE. Se elaboró también un registro con enlace directo al material elaborado. Desde el Servicio TIC (Servicio de Tecnologías de la Información y Comunicación) y el SARDT (Servicio de Adaptación de Recursos Didácticos y Tecnológicos) se revisa el cumplimiento de los criterios de accesibilidad.

Los materiales se clasifican por nivel educativo, materia, etiquetas (percepción visual, estimulación visual, estimulación auditiva, lectoescritura, Edico -Editor Científico ONCE-), y se redactan una breve descripción o instrucciones de uso.

Simultáneamente a este proyecto colaborativo de recogida de materiales se han planificado, desarrollado y potenciado diversos espacios en la Plataforma Moodle del CREDV-CRE ONCE Barcelona para que los especialistas de las áreas de especial dificultad (educación física, música, plástica, ciencias, matemáticas, inglés y tecnología) puedan subir pautas, orientaciones y materiales diversos que den soporte a los maestros itinerantes y también a los maestros de aula. 


\section{Desarrollo de nuevos procesos y metodologías de atención a las familias y alumnos más pequeños}

El proceso de atención telemática no es tan fácil cuando hablamos de familias y alumnos que todavía no están iniciados en el uso de las tecnologías, bien porque por edad no les corresponde, bien porque no disponen de los dispositivos ni de la tecnología necesaria, o porque todavía no tienen adquiridas las destrezas para ser autónomos.

En el caso de la atención temprana o de la educación infantil, las maestras de soporte, durante la pandemia, realizaron una atención telemática basada en el contacto telefónico, la conexión por WhatsApp (videoconferencia) o el uso de tabletas enfocando el entorno del alumno y compartiendo con las familias metodologías y estrategias de trabajo.

La realización de cuentos en formato de vídeo formó parte esencial de esta comunicación con los más pequeños.

\section{Reinvención de las actividades de formación}

En un escenario de confinamiento, además de establecer las pautas y metodologías de trabajo para continuar con la atención directa de los alumnos con discapacidad visual que realizaban un aprendizaje virtual desde sus casas, se debían planificar las actividades del CREDV-CRE ONCE Barcelona para el inicio del curso 2020-2021.

El reto fue doble: en primer lugar, convencer a todos los actores de que era necesario replantear todas las actividades del CREDV-CRE ONCE Barcelona para convertirlas en telemáticas en un posible escenario de aislamiento, y así continuar con el servicio que ofrece el Centro, aun a sabiendas de que un planteamiento virtual no es tan eficaz como el presencial; el segundo reto consistió en replantear y reinventar las múltiples actividades presenciales para convertirlas en telemáticas.

Las reuniones a distancia, discusiones, revisión de objetivos, propuestas en distintos escenarios, etc., fueron múltiples. Con la colaboración, esfuerzo e investigación de todos los profesionales fue posible reinventar las distintas actividades que el CREDV-CRE

Boix, S., Corbella, M.T., y Torres, F. (2021). Tecnología, creatividad y reinvención de actividades: las claves del éxito en la atención educativa a alumnos con discapacidad visual en tiempos de pandemia. RED Visual: Revista Especializada en Discapacidad Visual, 77, 240-263. https://doi.org/10.53094/LWAR1212. 
ONCE Barcelona venía realizando desde siempre, reconduciéndolas hacia un formato virtual.

\subsection{Cursos de formación TIC/Edico/BME dirigidos a alumnos}

A principios de septiembre se organizan diversos cursos de formación dirigidos a alumnos del CREDV-CRE ONCE Barcelona y su ámbito, a partir de segundo ciclo de primaria, con el objetivo de potenciar sus aprendizajes en el área de tiflotecnología e informática, matemáticas y música.

Tradicionalmente, estos cursos se impartían de forma presencial, durante un día completo (mañana y tarde), distribuyéndose los días en función de la herramienta tiflotécnica que usaba el alumno. Los alumnos se agrupaban por niveles y trabajaban tiflotecnología, ofimática, Edico, BME (siglas en inglés del Editor Braille Musical), etc. Todos los alumnos seguían una programación similar adaptada a sus conocimientos y necesidades.

La opción de tener a los alumnos conectados muchas horas era inviable, por lo que se optó por plantear la inscripción a los cursos por aprendizajes, con una duración máxima de una hora y media. De este modo, se ofertó a los alumnos una batería de diecinueve cursos, de diversas modalidades y niveles, a los cuales se podían inscribir según sus necesidades.

Así, en el área de matemáticas, se ofertó desde un curso de iniciación a Edico (para alumnos que todavía no tienen instalado el programa), hasta cuatro niveles distintos, partiendo de $5 .^{\circ}$ de primaria hasta bachillerato y CFGM (ciclos formativos de grado medio), y algunos específicos para trabajar Física y Química en la ESO y bachillerato.

En el área de informática, se ofrecieron aplicaciones de Google tales como Drive, Classroom, Docs, Slides, etc.; aplicaciones de Microsoft tales como Word, PowerPoint, Excel; acceso a PDF, y navegación por Internet. En el área de tiflotecnología, ZoomText y JAWS. En el área de música, iniciación al editor de partituras BME V2 (exposición teórica) y dos cursos a nivel práctico.

En la planificación se procuró que las formaciones por niveles no fueran en horarios coincidentes.

La demanda de cursos y formación superó nuestras expectativas y nos obligó a crear subgrupos de formación en una misma sesión. De este modo, por ejemplo, 
en el curso de Word, se crearon 3 clases telemáticas distintas atendidas por tres profesionales del Centro.

Es importante destacar que, con carácter previo a la realización de los cursos, se procuró, desde el Servicio TIC y en colaboración con los maestros de soporte, que el alumno inscrito conociera las herramientas de conexión (plataforma Zoom o Google Meet) y tuviese disponible el software de la formación (Word, Edico, etc.). Incluso se creó un vídeo tutorial explicativo para realizar la formación.

Los maestros de soporte conocían las necesidades en tiflotecnología e informática de sus alumnos, ya que, a finales del curso 2019-2020 y durante el confinamiento, rellenaron una encuesta sobre el nivel de aprendizaje TIC de sus alumnos, en la que, además de informar sobre las plataformas de aprendizaje que utilizaban, las editoriales, etc., valoraban, en una escala numérica, el conocimiento del alumno de todas las herramientas, como JAWS, ZoomText, Word, entre otras. De este modo, también pudieron orientar a sus alumnos para inscribirse en los cursos.

El resultado de la experiencia fue positivo. Evidentemente, hubo incidencias, algunas de las cuales se pudieron resolver al momento y otras no, pero sirvió para detectar necesidades de los alumnos que durante el curso se han ido resolviendo.

Hay que añadir que los maestros de soporte también asistieron a los cursos, siendo, en muchos casos, clave para ayudarlos en la formación. Algunos estaban conectados con el alumno también por TeamViewer en remoto.

Los materiales para trabajar en las sesiones se enviaron previamente por correo electrónico, se compartieron con un drive y también estaban disponibles en la plataforma Moodle del Centro. Asimismo, se crearon en WhatsApp diferentes grupos en que participaban los coordinadores de caso y los distintos profesionales que impartían los cursos, para así poder establecer una coordinación más eficaz y atender a las dificultades que pudieran presentarse durante la realización de estos.

\subsection{Cursos de formación dirigidos a profesionales}

Al igual que en el apartado anterior, era necesario reconvertir todas las formaciones y seminarios dirigidos a los profesionales de la escuela ordinaria para el curso 20202021.

Boix, S., Corbella, M.T., y Torres, F. (2021). Tecnología, creatividad y reinvención de actividades: las claves del éxito en la atención educativa a alumnos con discapacidad visual en tiempos de pandemia. RED Visual: Revista Especializada en Discapacidad Visual, 77, 240-263. https://doi.org/10.53094/LWAR1212. 
En estas formaciones sí que estaba claro que el formato sería telemático y que se podía contar con el soporte de la plataforma Moodle para realizarlas. Este entorno virtual de aprendizaje nos ha permitido realizar formaciones, inscribir a los participantes, abrir foros de participación, calificar, etc.

Todos los especialistas del Centro (plástica, música, educación física, inglés, ciencias, matemáticas, tiflotecnología e informática), además de los maestros de soporte que participaban en el curso Primer contacto con el Servicio Educativo CREDV-CRE ONCE Barcelona) han reinventado su formación.

La mayoría de los cursos y seminarios han contado con unas horas de conexión por videoconferencia (una o dos sesiones de hora y media) y el trabajo individual o en grupo de los participantes en la plataforma Moodle.

Esto ha conllevado la necesidad de adaptar los contenidos, las estrategias y metodologías de impartición de las charlas, aprender a utilizar el EVA (entorno virtual de aprendizaje) subir la documentación, motivar a los participantes mediante mensajes y foros, etc.

Esta experiencia se valora de forma positiva a pesar del trabajo y sobreesfuerzo de todos, ya que ha contribuido a ampliar el conocimiento tecnológico de los profesionales del Centro de diversas herramientas (edición de vídeos, edición en la plataforma Moodle, uso de foros, etc.).

\subsection{Otras actividades de formación de profesionales del Centro}

Otras actividades que se han reinventado han sido los Talleres de familias, el grupo de formación para profesionales del Centro Tecnología a la carta, los grupos de trabajo entre profesionales del Centro, como el de Creación y elaboración de materiales digitales accesibles...

Destacamos las siguientes.

\subsubsection{Grupo de trabajo para la realización de materiales digitales accesibles dirigido a maestros}

Este grupo se realizaba presencialmente con los miembros en la sala de ordenadores, y con dos miembros del Servicio TIC del Centro que aconsejan en la elaboración 
de materiales, y que, de alguna manera, hacen la función de formadoras. La mayor parte de los miembros se reorganizan en pequeños grupos para decidir un proyecto y elaborarlo de manera conjunta.

El trabajo online se lleva a cabo, primero, reuniendo a todos los miembros a través de una videoconferencia mediante la plataforma Zoom. Después, se forman grupos para realizar los trabajos. Cada uno de ellos crea su propia reunión con Google Meet, y en el chat general se anota el enlace. Salen de la reunión de Zoom general, para poder trabajar tranquilamente con los miembros de cada subgrupo y no interferir en las conversaciones. Antes de salir de la reunión conjunta, los subgrupos piden ayuda a las formadoras si la necesitan, y de esta manera «aparcamos» la conferencia general para entrar en las de cada grupo. Si alguno de ellos no ha solicitado ayuda, al tener anotadas en el chat sus reuniones, podemos acceder y hablar sobre el desarrollo del trabajo que están realizando y ayudar en caso necesario. Es como realizar un paseo virtual por las salas de cada subgrupo de trabajo. Antes de finalizar la sesión, todos volvemos a la reunión general de Zoom y ponemos en común algunas pinceladas del trabajo realizado.

Las formadoras nunca cerramos la sesión de Zoom general. Por ello, en algún momento ha sido necesario disponer de dos ordenadores para poder tener acceso al menos a dos sesiones a la vez, y es importante no salir, ya que cualquier anotación en el chat se pierde si se sale de la reunión. Hay que tener en cuenta silenciar el micro de la reunión en la que no se está participando activamente. En algún momento, se ha contado con la utilización de otro dispositivo, como tabletas o móviles, en el caso de no tener dos ordenadores.

Disponer de dos ordenadores facilita:

- Que las formadoras puedan compartir la pantalla para mostrar cómo realizar la adaptación del material, pues es en el ordenador donde se suelen tener instalados la mayoría de los programas para la elaboración de materiales accesibles.

- Que, a su vez, los maestros puedan compartir su pantalla con el objetivo de poder asesorarlos mejor en la realización de su trabajo.

Esta división en salas ahora también puede realizarse con la herramienta Zoom de forma automática. 


\subsubsection{Sesiones de Tecnología a la carta}

Son sesiones formativas dirigidas a profesionales en las que se informa de diversos temas, tanto en el área de tiflotecnología como de tecnología general, y que antes de la pandemia se realizaban de manera presencial. Ahora que se llevan a cabo de manera virtual, el aspecto positivo que hemos valorado es que ha permitido incorporar a un número mayor de profesionales de todo el territorio, llegando a muchas más zonas geográficas. Antes no era posible la asistencia presencial de estos profesionales, tanto por cuestiones de tiempo como organizativas, pero ahora, la conexión virtual permite no tener que cuadruplicar las sesiones (Barcelona, Gerona, Lérida y Tarragona) y, de esta forma, la formación que reciben los maestros llega para todos de igual manera. Se ha pasado de tener presencialmente una media de 20 profesionales en Barcelona, 8 en Gerona y 11 en Tarragona y Lérida, en distintas sesiones, a alcanzar ahora una media de 55 personas en cada sesión que se realiza. Hemos mantenido el uso de la plataforma Moodle para subir los contenidos que se explican en cada sesión, como ya se hacía cuando se realizaba de manera presencial.

Un elemento que hemos puesto en valor ha sido el de incrementar la elaboración de vídeos, para mostrar contenidos que permiten complementar la exposición. En el caso de que fallara algún aspecto técnico, al tener preparados los vídeos con anterioridad, estos se pueden utilizar, y el resultado está más asegurado. Por otro lado, se está elaborando una base de datos de vídeos para proporcionarlos como material de formación, tanto a futuros maestros, a maestros de aula y/o profesionales internos de nuestro Centro, como a los alumnos.

En algunas sesiones se ha introducido una herramienta nueva: el visor de documentos, que, conectado al ordenador, permite mostrar materiales físicos (líneas braille, teclados, etc.). Se conecta el visor, enfoca el material o los materiales y se realiza la explicación. Uno de los inconvenientes es que solo se dispone de uno de ellos en el Centro. Una posible alternativa es enfocar, con una cámara web externa, aquello que se quiere mostrar, pero es complejo encontrar la manera de sostenerla intentando enfocar correctamente el material $y$, al mismo tiempo, poder manejarlo mientras se está realizando la explicación. El proceso es el siguiente:

- La formación se imparte a través de la plataforma correspondiente, y se comparte nuestra pantalla para mostrar la presentación que tenemos preparada, los programas que queremos mostrar, etc.

Boix, S., Corbella, M.T., y Torres, F. (2021). Tecnología, creatividad y reinvención de actividades: las claves del éxito en la atención educativa a alumnos con discapacidad visual en tiempos de pandemia. RED Visual: Revista Especializada en Discapacidad Visual, 77, 240-263. https://doi.org/10.53094/LWAR1212. 
- En el momento de querer mostrar un material físico, dejamos de compartir la pantalla, se cambia la cámara del equipo a la cámara del visor que lo enfoca y se da la explicación correspondiente.

\subsection{Actividades del Servicio de Escolaridad Combinada}

Atendiendo a la normativa interna de la ONCE, el Servicio de Escolaridad Combinada/Compartida quedó regulado en la Circular 20/2006, de 24 de noviembre, de la Dirección de Educación. En el caso de la comunidad autónoma de Cataluña, se ha dado en llamar Servicio de Escolaridad Combinada, estableciéndose esa nomenclatura en el convenio firmado entre el Departamento de Educación de la Generalitat y la ONCE, con fecha de 5 de julio de 2018.

El Servicio de Escolaridad Combinada (SEC) es un servicio de apoyo a la educación inclusiva para aquellos alumnos cuyas circunstancias requieren de un refuerzo extraordinario para proseguir sus estudios con las mismas oportunidades que sus compañeros. Tiene dos vertientes: el SEC individual y el SEC grupal (actividades complementarias).

En el CREDV-CRE ONCE Barcelona, en el período prepandemia, el SEC individual (focalizado en las necesidades de cada alumno) se venía prestando de forma intensiva, acudiendo los alumnos al Centro para recibir, de parte de los profesionales dedicados a esta tarea, una intensificación de las técnicas, estrategias y contenidos, tanto curriculares como específicos derivados de su discapacidad visual, abordándose áreas como la atención psicológica, la atención emocional en grupo, el aprendizaje y consolidación del Braille, la educación física, la psicomotricidad, la música, la plástica, las matemáticas o la tiflotecnología.

La covid-19 hizo que, durante el período de confinamiento, hubiéramos de dejar de prestar este servicio, que se reemprendió a finales del primer trimestre del curso 2020-2021. No se inició antes, pues, dadas las especiales características del mismo, hubo de efectuarse una reflexión y planificación previas, planteándonos en qué aspectos (psicología, braille) se requería de presencialidad y si esta era posible; si los alumnos contaban con la suficiente autonomía como para efectuar por sí solos las conexiones telemáticas y el seguimiento de los contenidos o si, por el contrario, precisaban de la presencia del coordinador de caso u otra persona que diera soporte; si era factible o no trabajar a distancia en áreas como la educación física (que precisa 
de la interacción profesional/alumno para la realización de las actividades, además de contar con espacios acondicionados y con materiales específicos), la plástica (que requiere la manipulación física de material y las orientaciones in situ) o la música. Finalmente, se decidió que era necesario prestar este servicio, con las adaptaciones que ahora veremos.

Aunque la situación actual impide aún la normalidad, gracias a las posibilidades que brinda la tecnología se está logrando ofrecer un apoyo individualizado que, siendo distinto, da cobertura igualmente a las necesidades que alumnos y profesionales plantean.

El alumnado al que atendemos es, en este momento, aquel que no tiene resto visual funcional y debe adaptarse, en el ámbito tecnológico, al uso de los dispositivos con lectores de pantalla y con una metodología diferente a la que utilizan los alumnos sin discapacidad visual. Nuestro alumnado no hace uso del ratón y debe aprender a manejarse en braille, con audio y con teclado.

Encontramos alumnos cuya discapacidad visual se ha acrecentado en el último tiempo y otros que, aun teniéndola con anterioridad, precisan de la intensificación que antes referíamos por exigencias curriculares.

Se están efectuando sesiones de duración aproximada de sesenta minutos con carácter general, con periodicidad semanal o quincenal, dependiendo de los casos. Debido a las restricciones que obliga a imponer la pandemia, solo algunos de nuestros alumnos (para quienes la presencialidad resulta imprescindible, sea por sus necesidades o por las áreas de trabajo que debemos priorizar) están acudiendo al Centro, pudiendo permanecer en él un máximo de dos horas. En los demás casos, las sesiones son híbridas o del todo telemáticas.

Se utilizan plataformas como TeamViewer, JAWS Tandem, Zoom o Meet para las conexiones, acudiendo también a llamadas telefónicas comunes o a WhatsApp.

Se comparte la pantalla y el audio, por parte del formador o del propio alumno, y se realiza el control remoto del ordenador de este último si es necesario.

Se procura la capacitación y autonomía de los estudiantes para el acceso y realización de las actividades propuestas. Se cuenta, en situaciones en que dicha autonomía aún no es posible (debido a la edad o por otras circunstancias que concurren junto 
a la discapacidad visual), además de con algún tutor del centro educativo, con la inestimable colaboración de los maestros de apoyo del CREDV-CRE ONCE Barcelona, que se encuentran presencialmente junto a los alumnos.

Además, en el área de tiflotecnología (una de las más demandadas en el contexto actual), tras las clases impartidas, se elaboran apuntes que se remiten a los coordinadores de caso y a los alumnos si ya disponen de correo electrónico, para que les resulte más sencillo repasar y consolidar los contenidos dados. También, si se considera oportuno y previa autorización, se efectúa la grabación en audio de las sesiones, que se les hace llegar, pues entendemos que es una forma más de contribuir a su aprendizaje.

\subsubsection{Ejemplo de clase telemática para trabajar braille y plástica}

Una de las alumnas que atendemos, que cursa tercero de primaria, realiza semanalmente una sesión de 90 minutos. En el centro educativo se encuentra la coordinadora de caso, y por Meet se conectan dos profesionales del CREDV-CRE ONCE Barcelona para trabajar el braille y la plástica.

En braille, se trabaja la lectoescritura, aplicada, por ejemplo, a las matemáticas, y se proponen tareas cuya realización supervisa la maestra presencialmente. En plástica, se anticipan los materiales necesarios y, si no se dispone de ellos en el centro educativo, se preparan en nuestro centro y se hacen llegar al mismo a través de la coordinadora de caso. Se plantea la actividad por parte de la especialista, la alumna la ejecuta y, si precisa soporte, se lo da la maestra presencialmente.

Se pretende que la alumna pueda ejecutar por sí misma las tareas. La coordinadora de caso establece la conexión, procura que el enfoque de la cámara sea adecuado y solo interactúa si es preciso.

\subsubsection{Ejemplo de clase telemática para trabajar braille y tiflotecnología}

Con otro de los estudiantes, que cursa segundo de primaria, enfocamos la sesión, de 60 minutos, a la consolidación del braille y el uso del ordenador.

La tutora de aula establece la conexión vía Zoom y acude al alumno si existe algún problema. Se conectan simultáneamente dos profesionales del CRE, profesora de Braille y la ITB (Instructora en Tiflotecnología y Braille).

Boix, S., Corbella, M.T., y Torres, F. (2021). Tecnología, creatividad y reinvención de actividades: las claves del éxito en la atención educativa a alumnos con discapacidad visual en tiempos de pandemia. RED Visual: Revista Especializada en Discapacidad Visual, 77, 240-263. https://doi.org/10.53094/LWAR1212. 
Se conjuga la tiflotecnología con la pedagogía en el empleo del braille, usando la línea braille. La técnica en tiflotecnología establece conexión con JAWS Tandem y, a la vez, el alumno comparte pantalla y sonido en Zoom para que la maestra de Braille del CRE pueda seguir la sesión y participar en la misma.

Se preparan con anticipación materiales para la lectura y escritura en braille computerizado. La coordinadora de caso o la tutora disponen esos materiales en una carpeta específica del ordenador, y se trabaja con el alumno el acceso a los mismos. Se usan, pues, el ordenador y el braille de forma simultánea, introduciendo estrategias para el uso de los dispositivos, también con audio y teclado.

Se busca, a lo largo de las sesiones, aumentar la autonomía del alumno y la capacidad de comprensión y realización de las actividades propuestas.

\subsection{Actividades «complementarias» de alumnos con discapacidad visual}

Las actividades complementarias son un SEC que, a principios de cada curso, ofrece el Centro a todos los alumnos con discapacidad visual desde Educación Infantil hasta $4 .^{\circ}$ de la ESO, con el objetivo de trabajar juntamente con los profesionales del CREDVCRE ONCE Barcelona aquellas materias o aspectos de mayor dificultad en cada etapa educativa y también compartir entre iguales emociones, salidas, etc.

Durante este curso 2020-2021 también se han diseñado en un formato virtual, siempre con el objetivo de mantener el punto de encuentro del alumno con discapacidad visual y su «grupo de complementarias» del CREDV-CRE ONCE Barcelona.

De este modo, los alumnos de educación infantil son llamados a realizar actividades con sus padres quincenalmente, con artículos publicados en la página web Nodes. Con un formato de vídeo, las maestras de soporte exponen las actividades y, a través de un correo electrónico, los padres envían su respuesta. El centro de interés es El caracol Serafín.

- Cursa de cargols (Carrera de caracoles): enlace al vídeo https://youtu.be/k0w0IjolvxQ.

- Actividad de música: enlace al vídeo https://youtu.be/zidsnvK4fCI.

- Sesión de informática: enlace al vídeo https://youtu.be/s4ROIclh470.

- Animals (Animales): enlace al vídeo https://youtu.be/zU5HbV5Kv0c.

Boix, S., Corbella, M.T., y Torres, F. (2021). Tecnología, creatividad y reinvención de actividades: las claves del éxito en la atención educativa a alumnos con discapacidad visual en tiempos de pandemia. RED Visual: Revista Especializada en Discapacidad Visual, 77, 240-263. https://doi.org/10.53094/LWAR1212. 
Los alumnos de ciclo inicial y ciclo medio de primaria reciben mensualmente juegos y actividades por correo electrónico a través de sus padres.

Los de ciclo medio, mayoritariamente de baja visión, trabajan estas actividades usando Classroom.

Los alumnos de ciclo superior de primaria trabajan retos propuestos por los distintos especialistas de música, educación física, inglés, etc., a través de una carpeta de Google Drive compartida. Los realizan con ayuda de su maestro de soporte o de la familia y los comparten en la misma carpeta. Mensualmente, realizan una conexión por videoconferencia desde su escuela y comentan su experiencia.

Los alumnos de la ESO se conectan una tarde al mes y reciben una sesión de formación preparada por los especialistas del CREDV-CRE ONCE Barcelona, además de hablar de sus inquietudes.

Estas actividades se han diseñado tratando de utilizar las tecnologías que, durante el confinamiento, usaron los alumnos (Google Drive, Classroom, videoconferencia), con el objetivo de practicar con ellas y mejorar las destrezas en su uso.

En paralelo, las ITB realizan formaciones individualizadas para trabajar con aquellos alumnos que lo precisan.

En general la valoración de estas actividades es variada. Para los alumnos que tienen autonomía en el uso de las herramientas tiflotécnicas, es decir, a partir de ciclo superior de primaria, la valoración es positiva. En cursos inferiores no se tiene tanta respuesta en la realización de las actividades; aun así, en las reuniones de padres se explican y se anima a realizarlas. Se aprecia un cierto cansancio en la realización de actividades telemáticas.

\section{Metodologías de trabajo telemático con alumnos}

Tal y como se ha expuesto en anteriores apartados, frente a la relación de actividades formativas que se venían realizando presencialmente en nuestro centro de recursos, empezamos a buscar alternativas para llevarlas a cabo de forma virtual y encontrar la manera más adecuada para cada tipo de actividad, perfilando diversos tipos de metodologías con el alumnado. En general, podemos decir: 
- Se ha incrementado la realización de clases online, tanto grupales como individuales.

- Se ha potenciado el uso de los programas de control remoto además de los programas de reunión.

\subsection{Clases online grupales: ventajas y metodología}

La tecnología online permite atender a varios alumnos a la vez a pesar de estar dispersos geográficamente en el territorio. Sería más difícil atenderlos de manera presencial, sobre todo si se realizan las sesiones de formación de forma periódica. Con esto no se quiere decir que no consideremos importante la presencialidad, pero el hecho de tener una dispersión geográfica amplia, y debido a la edad temprana de los alumnos que no suelen aún desplazarse por sí solos, la mayoría necesitaban que sus familias los acompañasen al CRE. Esto implicaba una dificultad y, en algunos casos, no asistían a las sesiones de formación. Con esta modalidad, el alumno sí puede participar y aprender esos contenidos tan necesarios.

Metodología:

- Las sesiones online se realizan con el alumno en su centro escolar junto con el coordinador de caso, que está presencialmente a su lado.

- Esto permite que el maestro de soporte haga seguimiento del aprendizaje de su alumno y lo pueda ayudar en lo que sea necesario. Detecta posibles problemas que tiene el alumno, lo ayuda o nos lo hace saber para aplicar alguna solución o estrategia. Además, está al día del contenido que se le está impartiendo y puede potenciar la práctica en las sesiones que realizan juntos a lo largo del curso. En el caso de que el coordinador no pueda estar presencialmente, siempre se conectará también a la reunión para estar al día de los contenidos impartidos.

- Se utilizan algunas de las plataformas, siendo Google Meet o Zoom las más habituales. Por turnos, se pide a uno de los alumnos que comparta su pantalla mientras nosotras, las especialistas en tecnología, vamos impartiendo la formación. El resto de los alumnos sigue las instrucciones de la tarea, con la supervisión y, en caso necesario, ayuda de su maestro. 
- Previamente, se han enviado por correo electrónico los contenidos teóricos que se van a trabajar, así como los ejercicios que deben tener el día de la sesión para realizar la actividad y poder hacer todos a la vez el mismo ejercicio.

- Las valoraciones que se han realizado posteriormente con los coordinadores de caso han sido siempre positivas, de tal forma que se han organizado más sesiones.

Ejemplo de sesiones con cuatro alumnas de baja visión:

- Las alumnas son de cuatro poblaciones distintas de la provincia de Gerona, distantes entre ellas, quizás, hasta $80 \mathrm{~km}$.

- Cada MAE (maestro de apoyo a la enseñanza) está presencialmente con su alumna.

- Se imparte una formación en el uso de ZoomText.

- Conexión mediante Google Meet.

- Sesión de 1 hora y 30 minutos.

- Comparte pantalla una de las alumnas y se inicia la formación. La alumna debe ir haciendo el ejercicio y, posteriormente, se pasa el turno a la siguiente. Ello implica que su nivel de atención es alto, porque están preparadas para el momento en que les toque el turno.

\subsection{Clases online individuales: metodología e inconvenientes}

Se han realizado muchas sesiones de formación con la siguiente metodología: nos conectamos mediante la misma plataforma el coordinador de caso, el alumno y el profesional en tecnología.

Algunos de los objetivos de trabajo pueden ser:

- Resolver necesidades puntuales de formación del alumno derivadas en muchas ocasiones de su entorno escolar. 
- Consolidar aprendizajes específicos relacionados con sus propias herramientas de acceso al ordenador (JAWS, ZoomText, línea braille, etc.).

En algunas situaciones, al no haber ningún profesional a su lado, uno de los inconvenientes es que algunos problemas que se detectan no se llegan a poder resolver de manera telemática, y se ha de realizar presencialmente. Pero lo cierto es que la conexión ha ayudado a detectar necesidades y ha facilitado ser conscientes de ciertos problemas de manejo que el alumno presenta.

Si no es capaz de resolver algunas cuestiones en una situación controlada, en la que nuestro alumno está conectado solo, sin más compañeros, y con dos profesionales pendientes de él, cuando tenga que realizar las conexiones con todo su grupo-clase y un solo profesor, la probabilidad de que los problemas se repitan es muy alta, y su profesor de aula tampoco sabrá cómo ayudarle.

De alguna manera, nuestra formación es una práctica para que nuestros alumnos puedan enfrentarse a situaciones, pero dentro de su entorno escolar.

Ejemplo de clase online individual:

- Se expone el caso de una conexión que se realizó con una alumna de baja visión, su maestra de la ONCE y una de las ITB.

- La alumna utiliza la adaptación para ampliar, ZoomText, y se estaba realizando un repaso del uso de los atajos de teclado del programa. Cuando se le pedía a la alumna que ejecutara algunas combinaciones de teclas, no se activaba la función correspondiente. Por mucho que se intentó averiguar el problema, no fue posible. Se decidió ir presencialmente a su centro escolar para observar cómo trabaja la alumna. Se detectó algo muy simple. La alumna no utilizaba un orden adecuado en la combinación de las teclas y activaba antes otra función. Pero a partir de esta sesión, las posteriores se pudieron realizar sin problemas.

\subsection{Uso de programas de control remoto además de la plataforma de reunión}

Se han usado diversas metodologías: 
- Salir de la opción «Reunión» y conectarse en remoto (con el programa TeamViewer, o JAWS Tandem) para poder ayudar de manera más directa al alumno y manteniendo la comunicación por teléfono.

- Mantener con otro dispositivo (tableta, móvil) la misma reunión con Google Meet o Zoom para seguir con el contacto verbal y entrar en el ordenador con el control remoto.

En resumen, en más de una ocasión es necesario poder disponer de varios dispositivos para cubrir las diversas necesidades de formación e interacción con el alumno.

Todas las sesiones que se han realizado, tanto grupales como individuales, han sido valoradas positivamente tanto por los maestros como por los alumnos. Especialmente los alumnos de cursos superiores, que tienen más control de algunas herramientas y son mucho más autónomos, se sienten muy acompañados, sabiendo que tienen la posibilidad de recibir sesiones de formación de aquellos aspectos que necesitan para trabajar en sus centros respectivos.

Hay que decir que, aunque antes de la pandemia era una metodología que ya existía y se aplicaba en algunos casos, no era la primera opción escogida. Pero la realidad a la que nos hemos visto forzados ha mostrado que tiene muchos aspectos positivos, y, por ello, la mentalidad por parte de muchos profesionales sobre su valía está empezando a cambiar.

\section{Feedback de los usuarios (familia, alumno, profesional)}

Para los alumnos, el uso de la tecnología, aunque no exento de dificultades, ha tenido una repercusión positiva, en el ámbito educativo y también social o de ocio. Manifiestan, por ejemplo, que han tenido la oportunidad de trabajar con las herramientas de una forma sistemática e intensiva, lo cual ha favorecido un cada vez mejor desempeño en su uso. También, gracias a la tecnología, pueden ahora disfrutar de los materiales en formato digital de una forma más inmediata, pues, a nivel general, el contenido en papel ha pasado a un segundo plano. La tecnología les ha permitido conectarse y seguir las clases online como hubieran podido hacerlo de manera presencial, aunque para algunos han surgido inconvenientes en el momento de establecer esa conectividad. También utilizan los dispositivos tecnológicos para el

Boix, S., Corbella, M.T., y Torres, F. (2021). Tecnología, creatividad y reinvención de actividades: las claves del éxito en la atención educativa a alumnos con discapacidad visual en tiempos de pandemia. RED Visual: Revista Especializada en Discapacidad Visual, 77, 240-263. https://doi.org/10.53094/LWAR1212. 
ocio y el entretenimiento, así como para relacionarse con familia y amigos mediante asistentes de voz, correo electrónico u otros medios telemáticos. Se sienten apoyados por el CRE y agradecen la dedicación que se les ofrece.

Como aspectos menos positivos, algunos se refieren a los riesgos que puede suponer el uso de Internet, para los que quizás no están preparados, o al hecho de sufrir más cansancio visual (en caso de poder utilizar en mayor o menor medida un resto de visión).

Por su parte, las familias demuestran, en general, una gran satisfacción con el especial soporte que han recibido de parte de los profesionales del CRE en estos meses. Durante el confinamiento, tuvieron la posibilidad de estar continuamente al lado de sus hijos y la tecnología se ha convertido en aliada, pues existían familias con las que antes resultaba más difícil mantener el contacto y que, gracias a ella, han podido participar más de talleres y actividades organizados para ellas y/o para sus hijos. Mediante los talleres (por los que demuestran gran interés), están adquiriendo habilidades que les ayudan a apoyar a sus hijos. El hecho de interactuar mucho más con maestros y otros profesionales del Centro contribuye muy positivamente a que, del mismo modo que sus hijos, los padres también aprendan y refuercen los mecanismos para poder ayudarlos.

En lo que respecta a los profesionales, han sido capaces de adaptar su trabajo a las necesidades de los alumnos, de las familias y de los centros educativos, adquiriendo competencias y utilizando, en cada caso, las herramientas tecnológicas que se adecuaban mejor. Ha habido múltiples desafíos a los que hacer frente y también muchísima implicación y esfuerzo para lograr los resultados que se pretendían.

\section{Reflexión final}

Los alumnos con discapacidad visual grave son los que más necesitan anticipar el avance tecnológico para afrontar con la debida solidez los retos educativos y las competencias que les corresponde adquirir.

Para poder realizar un dictado en el procesador de textos, deben tener un dominio aceptable del teclado y, por tanto, se debe haber trabajado específicamente la mecanografía; y antes de imbuirse en la búsqueda de información en Internet, hemos debido 
anticipar con ellos la forma de explorar una página web, la estructura, los diferentes elementos que podemos encontrar en ella, planificando actividades en páginas web sencillas, con el uso de diccionarios en línea, etc. Es un largo proceso de aprendizaje.

De acuerdo con las Directrices de Educación de la Dirección General de la ONCE con relación a las competencias básicas en tiflotecnología y TIC, los alumnos con discapacidad visual en general y los ciegos en particular inician su formación en edades tempranas. Esto ha supuesto una ventaja, ya que han comenzado un aprendizaje sistemático de la tecnología antes de que el resto de sus compañeros debiera utilizarla de forma generalizada a causa de la pandemia. Aun así, encontramos que el uso de las herramientas no es, en algunos casos, tan ágil como demanda el contexto actual.

La covid-19 ha conllevado una expansión generalizada del aprendizaje telemático mediante herramientas educativas como ClassRoom, Google Docs, Google Forms (o sus análogas en Microsoft); plataformas como Alexia, Eleven...; el uso masivo del correo electrónico, o el empleo de libros digitales, entre otros. Partimos, en este sentido, de que, dentro de este amplio abanico, algunas de estas utilidades no son accesibles para los alumnos con baja visión o ceguera.

Desde marzo de 2020, siguiendo la dinámica general, en el CREDV-CRE ONCE BarceIona se han debido adaptar y modificar algunas de las fórmulas que hasta entonces se utilizaban: por ejemplo, aquellas que requerían de la presencialidad individual o grupal del alumnado, de las familias, de los profesionales.

La atención telemática ha demostrado, como veníamos analizando en párrafos anteriores, ser eficaz en muchas ocasiones. Permitiendo, por otra parte, un mejor aprovechamiento de los recursos, sin dejar de procurar la atención presencial requerida cuando las circunstancias (extremadamente fluctuantes a lo largo de estos meses) así lo han permitido.

Se optimiza la realización de las actividades propuestas, ya que se suprimen las barreras geográficas, se reducen los desplazamientos y se pueden agrupar los participantes en las formaciones, los asesoramientos, etc.

Con todo, encontramos que existen algunas dificultades en comparación con la atención presencial. En un contexto telemático es, sin duda, más complicado corregir posturas corporales o de los dedos, en el papel o en el teclado. 
A veces, si los espacios que ceden los centros educativos no son adecuados, puede existir una distorsión acústica que no favorece la comunicación.

La calidad de las redes y la velocidad de conexión, así como las características de los dispositivos, también pueden entorpecer el desarrollo ágil de las sesiones.

Sin duda alguna, los alumnos están a la altura de las circunstancias, esforzándose a diario por mejorar su capacitación, y también desde el CREDV-CRE ONCE Barcelona se están poniendo todos los medios al alcance para contribuir de forma activa a ese cometido.

Silvia Boix Hernández. Instructora en Tiflotecnología y Braille. Centro de Recursos Educativos de la ONCE en Barcelona. Gran Via de les Corts Catalanes, 394; 08015 Barcelona (España). Correo electrónico:sbh@once.es.

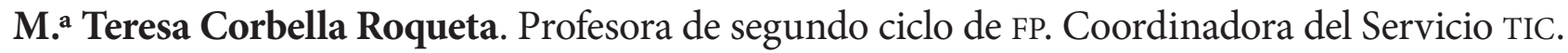
Centro de Recursos Educativos de la ONCE en Barcelona. Gran Via de les Corts Catalanes, 394; 08015 Barcelona (España). Correo electrónico: mtcr@once.es.

Francisca Torres Portero. Instructora en Tiflotecnología y Braille. Centro de Recursos Educativos de la ONCE en Barcelona. Gran Via de les Corts Catalanes, 394; 08015 Barcelona (España). Correo electrónico: ftpor@once.es. 